\title{
Numerical Analysis of Macro-Scale Mechanical Behaviors of 3D Orthogonal Woven Composites using a Voxel-Based Finite Element Model
}

\author{
Song Yu ${ }^{1} \cdot$ Diantang Zhang ${ }^{1} \cdot K_{\text {Kun }}$ Qian $^{1}$
}

Received: 7 February 2018 / Accepted: 8 March 2018 / Published online: 23 July 2018

(C) Springer Science+Business Media B.V., part of Springer Nature 2018

\begin{abstract}
A study is conducted with the aim of developing voxel-based finite element method related to the whole fiber distribution for predicting the macro-mechanical properties of 3D orthogonal woven composites. For the rationality of this model, multi-scale finite element method, which is on the basis of the surface and interior representative volume cells, and digital image correlation tests are carried out. The results show that the proposed voxel-based finite element method is capable of precisely calculating the macro-level properties of 3D orthogonal woven composites, validated by the comparison the mechanical behaviors as well as the full-field strain fields.
\end{abstract}

Keywords 3D orthogonal woven $\cdot$ Hybrid composites $\cdot$ Mechanical properties $\cdot$ Voxel-based finite element method $\cdot$ Macro-scale finite element method

\section{Introduction}

Owing to the comparable capability of weight reduction with excellent mechanical properties, threedimensional (3D) woven composites as structural components are being used increasingly for the space and aircraft applications, such as rag brace, propeller blades and trailing arms etc. [1-3] A major advantage of 3D woven technology to fabricate such complex-shaped components is the near-net shape capacity. However, the full-field fiber distribution of the obtained preform is marked heterogeneous. This has important impact on the macro-mechanical properties of the composite materials. The current macroscopic-like point of view is usually adopted by idealizing the heterogeneous phases as the homogeneous media [4]. In fact, this limitation reduced seriously the accuracy for predicting the mechanical behavior of macro composites. Thus, a more effective method related to the full-field fiber distribution is needed in order to provide a powerful tool for a better design.

Kun Qian

qiankun@jiangnan.edu.cn

1 Key Laboratory of Eco-Textiles, Ministry of Education, Jiangnan University, Wuxi 214122, China 
As reviewed in the previous reports, the current popular macro-mechanical characterization to the study of woven composites can be largely belong to multi-scale method $[5,6]$. Generally, the multi-level structure of woven composites can be divided into three scales: micro-, meso- and macro-scale, which correspond to matrix impregnated yarn, representative volume cell (RVC), and whole component, respectively. Homogenized macro-scale method can be employed to evaluate the stiffness, strength, damage initiation/evolution from the micro-scale and meso-scale step-by-step. For instance, Kwon YW et al [7] discussed the thermomechanical analysis of refractory woven fabric composites using a multi-scale analysis technique. Bacarreza et al. [8] developed a semi-analytical homogenization method to predict the progressive degradation of plain woven composites. Also, the failure of the open hole tension specimens was simulated. Lu et al [9] studied the stress-strain and progressive failure behaviors of $2.5 \mathrm{D}$ woven composites subjected to the axial tensile loading by using a two-step multi-scale method. Recently, Dai et al. [10] presented a detailed mosaic macro model to predict the elastic and damage progressive process of 3D woven composites. Bassam ES et al [11] developed multi-scale modeling of strongly heterogeneous 3D woven composite structures based on a spatial Voronoi tessellation. The numerical results showed that the predicted stiffness and surface strain agree well with the experimental ones. Wang et al. [12] proposed a multi-scale modeling to study the damage responses of $3 \mathrm{D}$ orthogonal woven composites subjected to the quasi-static and high strain rate compressions. This multi-scale model mainly includes [1] micro/meso/macro models with periodic boundary conditions for homogenizing the heterogeneous constituent system into RVC, and [2] a macro-scale rate dependent plasticity model combined with the critical damage area failure theory. Unfortunately, the macro-scale properties of homogenization woven composite, based on the basis of the result of meso-scale finite element solid models, can not reflect precisely the effect of the full-field fiber distribution.

In order to overcome the above difficulties, some novel macro-scale methods need to be developed. In our previous paper [13], fiber embedded matrix method related to the full-field fiber distribution is proposed to evaluate the macro-mechanical properties of $3 \mathrm{D}$ textile composites. The present work is aimed at establishing a simple and accurate analytical method to calculate the macroscopic mechanical properties of 3D orthogonal woven composites using a voxel-based finite element model (VFEM). Firstly, the detailed microstructures of two types of $3 \mathrm{D}$ orthogonal woven composites are established. Subsequently, voxel-based finite element models, with different mesh sizes, are proposed. Meanwhile, for comparison, multi-scale finite element model (MFEM), based on the surface and interior RVCs, are also presented. Then a careful experimental details including the composite specimen preparation and the digital image correlation (DIC) tests are described. Furthermore, the predicted results of VFEM are shown and compared with those of MFEM and experiment. Finally, some valuable conclusions are summarized.

\section{Microstructures of 3D Orthogonal Woven Composites}

The weave style considered in this paper is 3D orthogonal woven composites, as shown in Fig. 1. The warp and weft are oriented along the $x$-direction and y-direction, respectively. The $Z$ yarns interlocks with the weft layers through the thickness (z-direction). Also, the warp and $Z$ yarns arranges alternately with a certain ratio of 1:1 along the $\mathrm{X}$-direction. The preform structural parameters which are also known as manufacturer specified parameters are the 


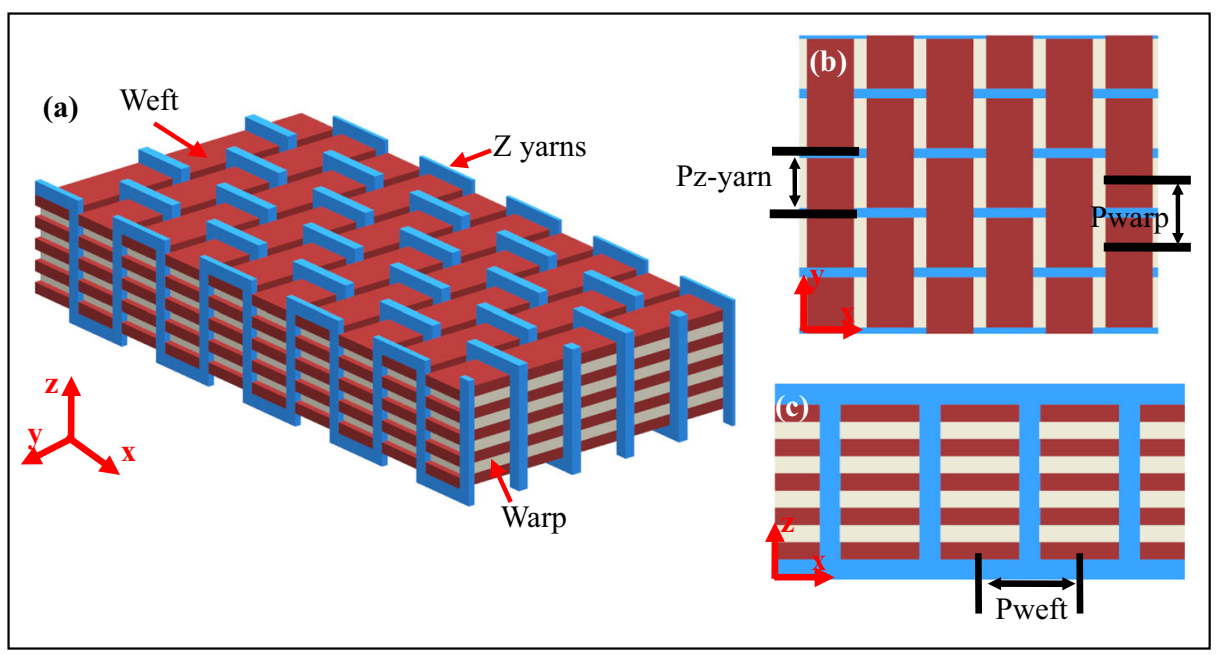

Fig. 1 Schematic diagram of 3D orthogonal woven composite

number of layers $(n)$, the warp density $\left(P_{\text {warp }}\right)$, the weft density $\left(P_{\text {weft }}\right)$, the $\mathrm{Z}$ yarn density $\left(P_{z-}\right.$ yarn $)$, the warp fineness $\left(\right.$ Tex $\left._{\text {warp }}\right)$, the weft fineness $\left(\right.$ Tex $\left._{\text {weft }}\right)$ and the $\mathrm{Z}$ yarn fineness $\left(\right.$ Tex $_{z-}$ yarn). These detailed parameters are summarized in Table 1.

For textile composites, the accuracy of the mechanical behavior simulation is highly dependent upon their actual microstructures [14]. The analytical geometry model proposed in this paper takes into account the cross-section and paths of yarns. It becomes known that a composite is an extremely complicated structure for the mechanical analysis. There exist several structural levels from yarns to preform and eventually to the composite. In each level, there are many geometrical and mechanical variables which control or influence the final composite behaviors. Therefore, this paper retains the essence of the physics but avoid unnecessary complexity so as to make our model $\mathrm{r}$ more user-friendly. On the basis of the microscopic image analysis (Fig. 2a and b), the following assumptions should be provided.

(1) For both carbon/epoxy composite and carbon/Kevlar/epoxy composite, the ideal crosssection shape of warp, weft and $\mathrm{Z}$ yarn, respectively, are consistent.

(2) The section of surface weft is an ensemble of ellipse and rectangular, while the section of interior weft are rectangular, as shown in Fig. $2 \mathrm{~d}$.

(3) Both the section of warp and weft are rectangular (see Fig. 2c and e). Also, warp and weft remain straight straighten.

Table 1 The detailed manufacturing parameters of the considered preform

\begin{tabular}{llllll}
\hline Preform type & & Yarn type & $\begin{array}{l}\text { Yarn density } \\
\text { (tows /cm) }\end{array}$ & $\begin{array}{l}\text { Yarn } \\
\text { fineness(Tex) }\end{array}$ & $\begin{array}{l}\text { Number of } \\
\text { layers }\end{array}$ \\
\hline Carbon & Warp & carbon & 5 & 800 & 4 \\
& Weft & carbon & 5 & 800 & 4 \\
Carbon/Kevlar & Z yarn & carbon & 5 & 400 & 400 \\
& Warp & carbon & 5 & 800 & 158 \\
\hline
\end{tabular}




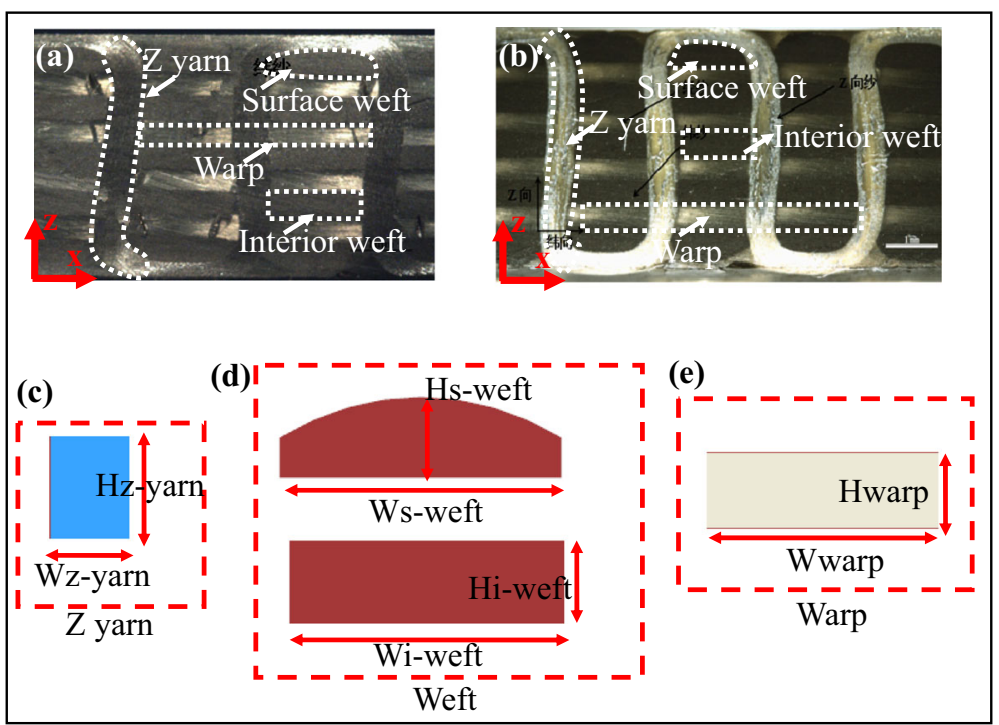

Fig. 2 Detailed structural information of 3D orthogonal woven composite: (a) real yarn cross sections of carbon/ epoxy composites, (b) real yarn cross sections of carbon/kevlar/epoxy composites, (c) ideal shapes of Z yarn, (d) ideal shape of weft, (e) ideal shape of warp

The geometrical parameters of yarns can be expressed as

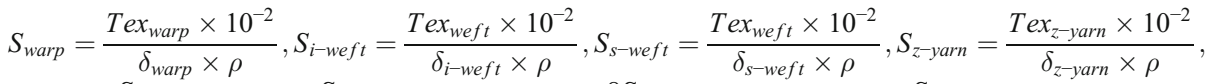

$$
\begin{aligned}
& H_{\text {warp }}=\frac{S_{\text {warp }}}{W_{\text {warp }}}, H_{i-\text { weft }}=\frac{S_{i-\text { weft }}}{W_{i-\text { weft }}}, H_{s-\text { weft }}=\frac{8 S_{s-\text { weft }}}{4 W_{s-\text { weft }}+\pi W_{s-\text { weft }}}, H_{z-\text { yarn }}=\frac{S_{z-\text { yarn }}}{W_{z-\text { yarn }}}
\end{aligned}
$$

Where $S_{\text {warp }}\left(\mathrm{mm}^{2}\right), S_{i-\text { wefi }}\left(\mathrm{mm}^{2}\right), S_{s-\text { wefi }}\left(\mathrm{mm}^{2}\right)$ and $S_{z-\text { yarn }}\left(\mathrm{mm}^{2}\right)$ are the cross-sectional area of warp, interior weft, surface weft and Z-yarn, respectively.

$\operatorname{Tex}_{\text {warp }}($ tex $), \operatorname{Tex}_{\text {wefi }}(t e x)$ and $\operatorname{Tex}_{z-\text { yarn }}($ tex $)$ are warp fineness, weft fineness and Z-yarn fineness, respectively.

$\rho\left(\mathrm{g} / \mathrm{cm}^{3}\right)$ is the density of carbon/Kevlar fiber.

$\delta_{\text {warp }}, \delta_{i-\text { weft }}, \delta_{s-\text { weft }}$ and $\delta_{z-\text { yarn }}$ are fiber volume fraction of warp, weft and $\mathrm{Z}$ yarn, respectively. Here, it should be noted that the cross-sectional shape of warp, interior weft and Z yarn is rectangular, $\delta_{\text {warp }}=\delta_{i-\text { weft }}=\delta_{z-\text { yarn }}=0.785$ [16]. However, the cross-sectional shape of surface weft is an ensemble of ellipse and rectangular. Here, $\delta_{s-\text { weft }}=0.76$ [15].

$H_{\text {warp }}, H_{i-\text { weft }}, H_{s-\text { weft }}$ and $H_{z-\text { yarn }}$ is the thickness of single warp, interior weft, surface weft and Z-yarn, respectively.

$W_{\text {warp }}, W_{i-\text { weft }}, W_{s-\text { weft }}$ and $W_{z-\text { yarn }}$ is the width of single warp, interior weft, surface weft and Z-yarn, respectively.

According to Fig. 1, the geometrical relationship can be expressed as.

$$
\begin{aligned}
& \frac{10}{P_{\text {warp }}}=\frac{10}{P_{z-\text { yarn }}}=W_{\text {warp }}+H_{z-\text { yarn }}, \frac{10}{P_{\text {weft }}}=W_{i-\text { weft }}+W_{z-\text { yarn }}=W_{s-\text { weft }}+W_{z-\text { yarn }}, \\
& T=n \times H_{\text {warp }}+(n-1) \times H_{i-\text { weft }}+2 \times H_{s-\text { weft }}
\end{aligned}
$$


By calculating, the corresponding geometrical parameters of carbon/epoxy and carbon/ Kevlar/epoxy 3D orthogonal woven composites are summarized in Table 2. Also, the mesoscale geometric models of carbon/epoxy and carbon/Kevlar/epoxy 3D woven composite, respectively, are illustrated in Fig. 2.

\section{Analytical Model}

As shown in Fig. 3, an analytical framework of mechanical property is presented. Two methods, voxel-based finite element model (VFEM) and multi-scale finite element method (MFEM) are proposed to predict the macro-scale elastic analysis of 3D orthogonal woven composites. The detailed analysis is described in the subsequent section.

\subsection{Materials Properties of Matrix-Impregnated Fiber Bundles}

In this paper, the yarn is carbon T700-6 K, carbon T700-12 K and Kevlar, and the matrix is epoxy resin (TDE 86). The mechanical properties of component materials are summarized in Table 3. For 3D orthogonal woven composites, yarns are thought to be the matrix-impregnated fiber bundles, which are assumed to be transversely isotropic. For the matrix-impregnated fiber bundles, the stress increments in the constituent fiber and matrix can be correlated by the bridging matrix as follows [16]:

$$
\left\{d \sigma_{i}^{m}\right\}=\left[A_{i j}\right]\left\{d \sigma_{j}^{f}\right\}
$$

Where

$$
\left\{d \sigma_{i}\right\}=\left\{d \sigma_{11}, d \sigma_{22}, d \sigma_{33}, d \sigma_{23}, d \sigma_{12}, d \sigma_{13}\right\}^{T}
$$

$\left\{d \sigma_{i}^{m}\right\}$ and $\left\{d \sigma_{i}^{f}\right\}$ represent the matrix stress increments and the fiber stress increments, respectively. $\left\lfloor A_{i j}\right\rfloor$ is the bridging matrix, which is expressed by:

$$
\left[A_{i j}\right]=\left[\begin{array}{cccccc}
a_{11} & a_{12} & a_{13} & 0 & 0 & 0 \\
a_{21} & a_{22} & a_{23} & 0 & 0 & 0 \\
a_{31} & a_{32} & a_{33} & 0 & 0 & 0 \\
0 & 0 & 0 & a_{44} & 0 & 0 \\
0 & 0 & 0 & 0 & a_{55} & 0 \\
0 & 0 & 0 & 0 & 0 & a_{66}
\end{array}\right]
$$

Table 2 Geometrical parameters of 3D orthogonal woven composites

\begin{tabular}{lllllllll}
\hline Composite type & $\begin{array}{l}H_{\text {warp }} \\
(\mathrm{mm})\end{array}$ & $\begin{array}{l}H_{i-\text { weft }} \\
(\mathrm{mm})\end{array}$ & $\begin{array}{l}H_{s-\text { weft }} \\
(\mathrm{mm})\end{array}$ & $\begin{array}{l}H_{z-\text { yarn }} \\
(\mathrm{mm})\end{array}$ & $\begin{array}{l}W_{\text {warp }} \\
(\mathrm{mm})\end{array}$ & $\begin{array}{l}W_{i-\text { weft }} \\
(\mathrm{mm})\end{array}$ & $\begin{array}{l}W_{s-\text { weft }} \\
(\mathrm{mm})\end{array}$ & $\begin{array}{l}W_{z-\text { yarn }} \\
(\mathrm{mm})\end{array}$ \\
\hline Carbon/epoxy & 0.31 & 0.48 & 0.75 & 0.17 & 1.83 & 0.65 & 0.48 & 1.51 \\
Carbon/Kevlar/epoxy & 0.29 & 0.50 & 0.76 & 0.08 & 1.91 & 0.65 & 0.50 & 1.50 \\
\hline
\end{tabular}




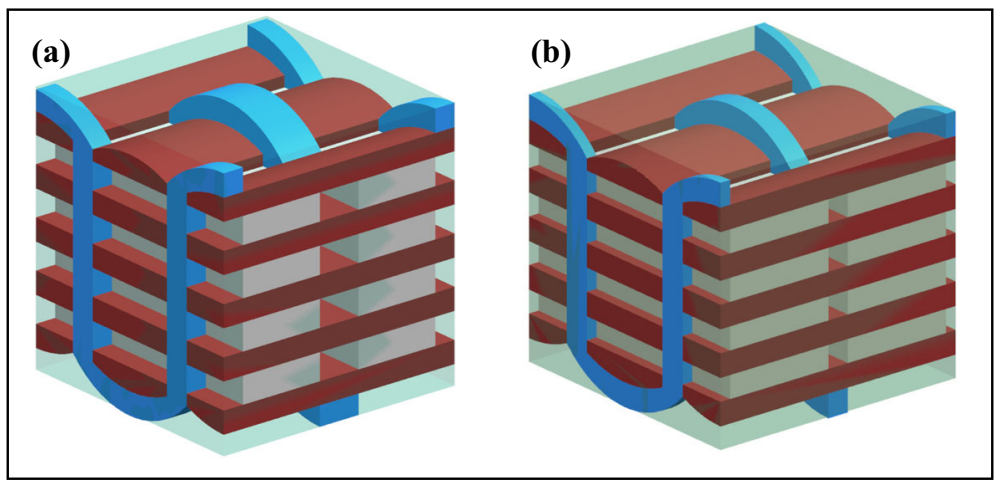

Fig. 3 Meso-scale RVC of 3D orthogonal woven composite, (a) carbon/epoxy, (b) carbon/kevlar/epoxy

Where

$$
\left\{\begin{array}{l}
a_{11}=E^{m} / E_{11}^{f} \\
a_{12}=a_{13}=\left(S_{12}^{f}-S_{12}^{m}\right)\left(a_{11}-a_{22}\right) /\left(S_{11}^{f}-S_{11}^{m}\right) \\
a_{21}=a_{31}=a_{23}=a_{32}=0 \\
a_{22}=a_{33}=1 / 2\left(1+E^{m} / E_{22}^{f}\right) \\
a_{44}=1 / 2\left(1+G^{m} / G_{12}^{f}\right) \\
a_{55}=a_{66}=1 / 2\left(1+G^{m} / G_{12}^{f}\right)
\end{array}\right.
$$

In the above formulas, $E^{m}$ and $G^{m}$ are the elastic and shear modulus of the matrix, respectively. $E_{11}^{f}, E_{22}^{f}$ and $G_{12}^{f}$ are the longitudinal, transverse and shear modulus of the fiber, respectively.

When the bridge matrix is given, the compliance matrix for the matrix-impregnated fiber bundles is defined as follows:

$$
\left[S_{i j}\right]_{n}=\left(V_{f}^{*}\left[S_{i j}^{f}\right]+V_{m}^{*}\left[S_{i j}^{f}\right]\left[A_{i j}\right]\right)\left(V_{f}^{*}[I]+V_{m}^{*}\left[A_{i j}\right]\right)^{-1}
$$

Where $\left[S_{i j}^{f}\right]$ and $\left[S_{i j}^{m}\right]$ represent the fiber and matrix compliance matrices, respectively. [I] is a unit matrix. $V_{f}^{*}$ and $V_{m}^{*}$ are the fiber and matrix volume fractions of the matrix-impregnated fiber bundles, respectively, and the relation is $V_{f}^{*}+V_{m}^{*}=1$.

Table 3 Mechanical parameters of constituents

\begin{tabular}{lllll}
\hline Type & Mechanical parameters & Carbon T700-12 K & Carbon T700-6 K & Kevlar \\
\hline \multirow{2}{*}{ Yarn } & Longitudinal modulus $E_{11}^{f}(\mathrm{GPa})$ & 232 & 221 & 156 \\
& Transverse modulus $E_{22}^{f}(\mathrm{GPa})$ & 15 & 13.8 & 90.9 \\
& Longitudinal poisson ratio $\nu_{12}^{f}$ & 0.28 & 0.2 & 0.19 \\
& Longitudinal shear modulus $G_{12}^{f}(\mathrm{GPa})$ & 24 & 9 & 17.3 \\
& Transverse shear modulus $G_{23}^{f}(\mathrm{GPa})$ & 5.03 & 4.93 & 38.19 \\
& & Epoxy & & \\
Matrix & Moulus $E^{m}(\mathrm{GPa})$ & 3.5 & & \\
& Poisson ratio $\nu^{m}$ & 0.35 & & \\
\hline
\end{tabular}


Furthermore, the relation between the compliance matrix and the engineering constants of the matrix-impregnated fiber bundles can be expressed by

$$
[S]=\left[\begin{array}{cccccc}
\frac{1}{E_{L}} & \frac{v_{L T}}{E_{T}} & \frac{v_{L Z}}{E_{Z}} & 0 & 0 & 0 \\
\frac{v_{L T}}{E_{L}} & \frac{1}{E_{L}} & \frac{v_{T Z}}{E_{Z}} & 0 & 0 & 0 \\
\frac{v_{L Z}}{E_{L}} & \frac{v_{T Z}}{E_{T}} & \frac{1}{E_{Z}} & 0 & 0 & 0 \\
0 & 0 & 0 & \frac{1}{G_{T Z}} & 0 & 0 \\
0 & 0 & 0 & 0 & \frac{1}{G_{L T}} & 0 \\
0 & 0 & 0 & 0 & 0 & \frac{1}{G_{L Z}}
\end{array}\right]
$$

By calculating, the mechanical properties of the matrix-impregnated fiber bundles are listed in Table 4.

\subsection{Method 1: Voxel-Based Finite Element Model (VFEM)}

For traditional meso-scale finite element solid models, the discrete mesh are quite tedious owing to the complex yarn architecture and the cross section shapes. A non-uniform element size and distorted elements are most probably obtained, which not only affects significantly the computational efficiency of the analysis, but also results in the inaccurate stress and strain distribution [17, 18]. Voxel meshing is an advanced method to generate meshes of any kind of textile architecture. The advantages of this methodology for modeling $3 \mathrm{D}$ orthogonal woven composites are [1] quick and automatic generation of regular 3D grid of voxel meshes [2]; assigning automatically the material properties. Several authors [19-21] has applied voxelbased finite element method to calculate the damage initiation, progression and homogenized thermo-elastic properties of 3D woven composites. The framework is summarized in Fig. 4.

In this study, voxel-based finite element method is developed to study the macro-mechanical properties of 3D orthogonal woven composites. The detailed full-scale model of $100 \mathrm{~mm} \times$ $20 \mathrm{~mm} \times 4.2 \mathrm{~mm}$ is built, as shown in Fig. 5. The three considered elements of $N_{x} \times N_{y} \times N_{z}$ are generated with TexGen (see Fig. 6). Table 5 summarizes the results of the experimental and predicted fiber volume fractions. It can be clearly seen that a general problem of voxel mesh is

Table 4 Predicted elastic constants of matrix impregnated fiber bundles using bridge matrix method

\begin{tabular}{lllll}
\hline & $\begin{array}{l}\text { Carbon/epoxy } \\
(\mathrm{T} 700-12 \mathrm{~K}, \delta=0.785)\end{array}$ & $\begin{array}{l}\text { Carbon/epoxy } \\
(\mathrm{T} 700-12 \mathrm{~K}, \delta=0.76)\end{array}$ & $\begin{array}{l}\text { Carbon/epoxy } \\
(\mathrm{T} 700-6 \mathrm{~K})\end{array}$ & Kevlar/epoxy \\
\hline$E_{L}(\mathrm{GPa})$ & 173.79 & 168.37 & 165.59 & 117.11 \\
$E_{T}(\mathrm{GPa})$ & 10.7 & 10.32 & 10.15 & 8.88 \\
$E_{Z}(\mathrm{GPa})$ & 10.7 & 10.32 & 10.15 & 8.88 \\
$\nu_{L T}$ & 0.41 & 0.39 & 0.3 & 0.5 \\
$\nu_{L Z}$ & 0.41 & 0.39 & 0.3 & 0.5 \\
$\nu_{T Z}(\mathrm{GPa})$ & 0.44 & 0.44 & 0.42 & 0.32 \\
$G_{L T}(\mathrm{GPa})$ & 8.12 & 7.47 & 5.27 & 4.26 \\
$G_{L Z}(\mathrm{GPa})$ & 8.12 & 7.47 & 5.27 & 4.26 \\
$G_{T Z}(\mathrm{GPa})$ & 3.70 & 3.58 & 3.59 & 3.36 \\
\hline
\end{tabular}




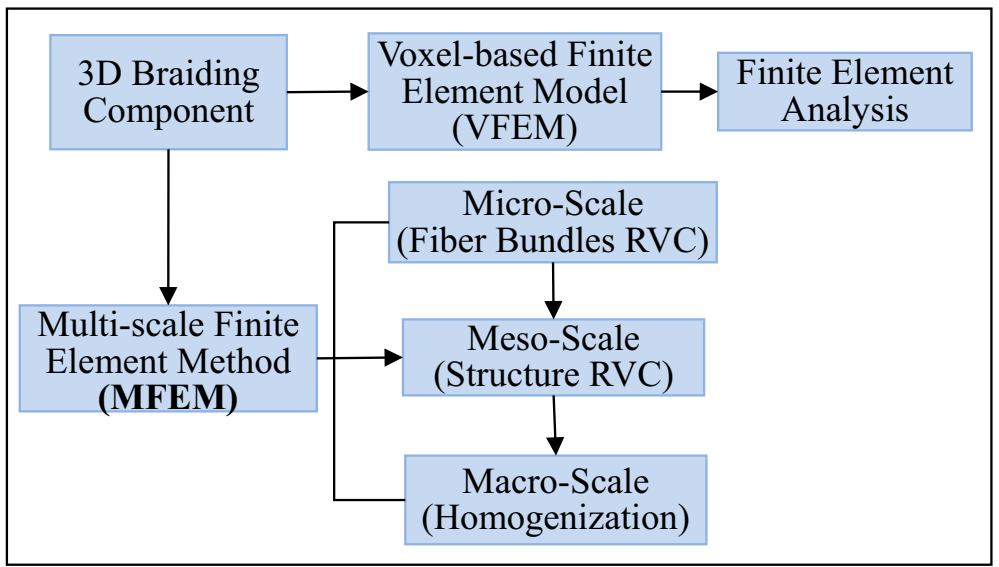

Fig. 4 Analytical framework of macro-mechanical property

that non-orthogonal interfaces appear stepped in nature. This phenomenon cannot be avoided, but its influence can be effectively reduced by refining the voxel mesh size. However, when the mesh sizes are much small, the computational time and cost increase greatly. Also, another

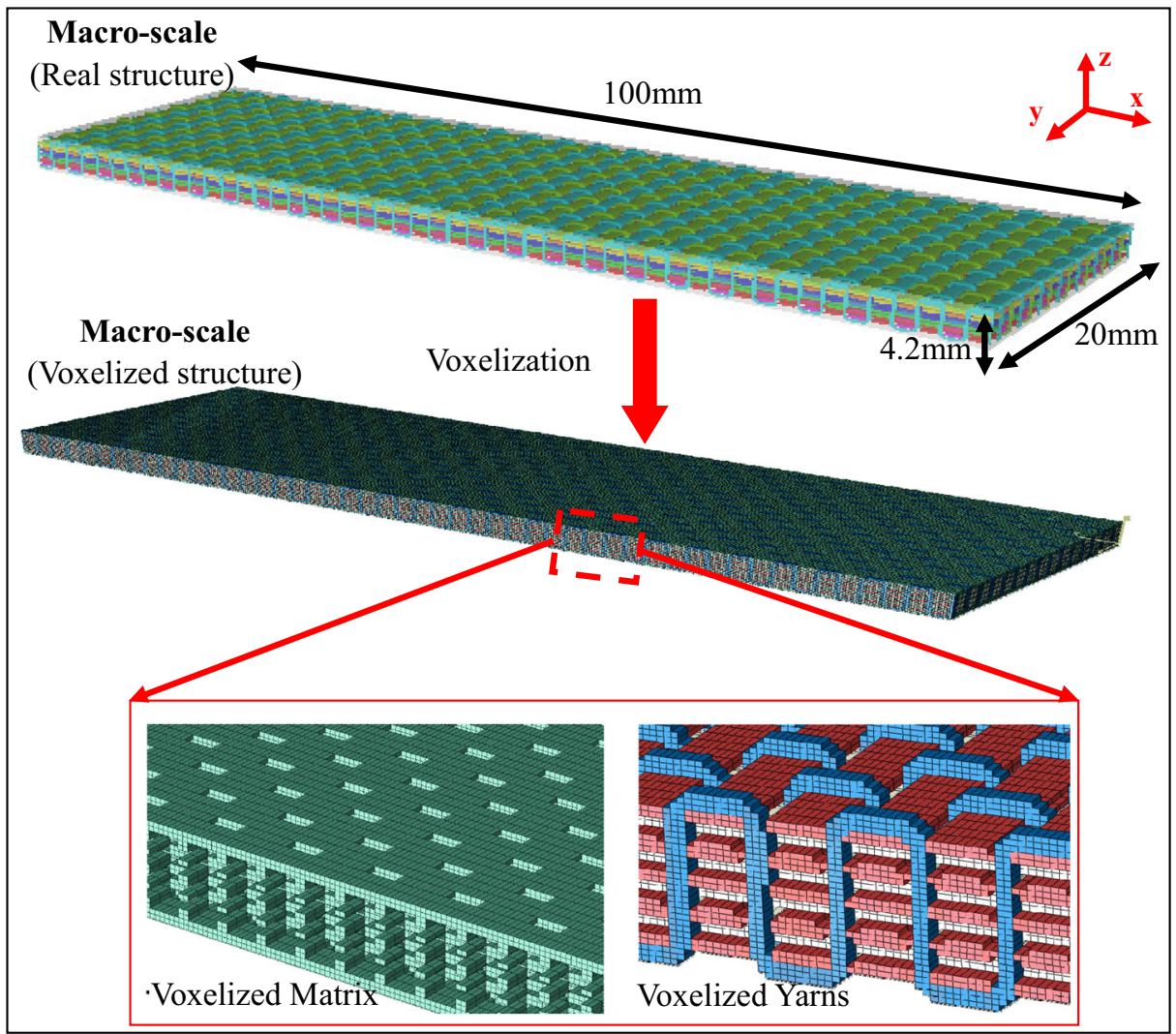

Fig. 5 Voxel-based model of macro component 


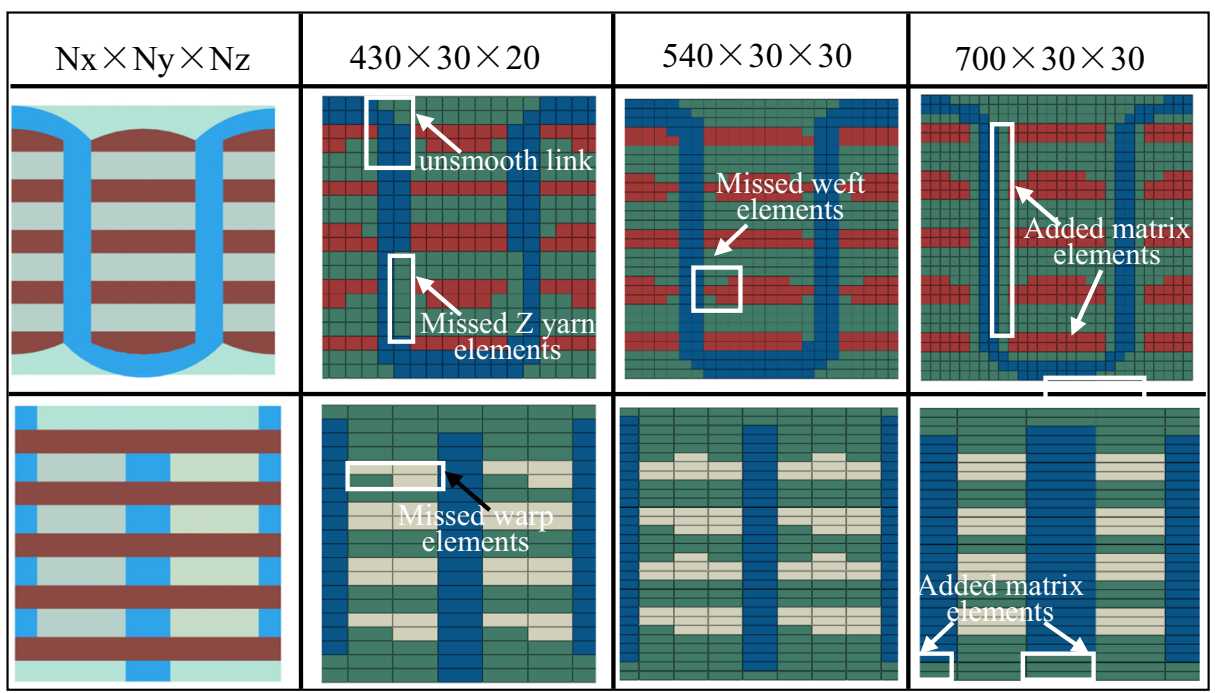

Fig. 6 Voxel-based model with different mesh sizes

problem of adjusting element size of the voxels is that there may be added matrix elements between yarns, missing yarn elements and unsmooth link etc (see Fig. 6). The effect of the mesh number on the mechanical properties will be discussed in the following section.

Once the voxel mesh of the macro-scale model has been obtained, it is necessary to apply the correct boundary conditions as well as the materials directions, and then imported into a finite element program (Abaqus) to simulate the macro elastic properties of the material.

\subsection{Method 2: Multi-Scale Finite Element Method (MFEM)}

Figure 7 presents the framework of multi-scale finite element model of 3D orthogonal woven composites. The MFEM is carried out using Abaqus/Standard software. The most basic step in developing MFEM is to predict the elastic constants of the matrix-impregnated fiber bundles. Considering the periodic fiber arrays, a hexagonal RVC instead of the matrix-impregnated fiber bundles is used in this paper (see Fig. 7).

Next, the effective mechanical properties of the matrix-impregnated fiber bundles are passed to the meso-scale solid model. Here, it should be noted that meso-scale RVC, which serves as a bridge linking the micro-scale analysis and macro-scale analysis, is capable to efficiently capture the effects of complicated yarn architectures. For 3D orthogonal woven composites, there are two types of RVCs, interior RVC and surface RVC. By applying the

Table 5 Fiber volume fraction of varying voxel mesh models

\begin{tabular}{llllll}
\hline Composite type & $N_{x} \times N_{y} \times N_{z}$ & Warp (\%) & Weft (\%) & Z yarn (\%) & Total (\%) \\
\hline Carbon/epoxy & $430 \times 30 \times 20$ & 21.42 & 23.37 & 7.95 & 52.74 \\
& $540 \times 30 \times 30$ & 23.53 & 23.59 & 6.35 & 53.47 \\
& $700 \times 30 \times 30$ & 23.94 & 23.52 & 6.89 & 54.35 \\
Carbon/Kevlar/epoxy & Experimental & - & - & - & 53.69 \\
& $540 \times 30 \times 30$ & 25.32 & 24.26 & 5.09 & 54.67 \\
& Experimental & - & - & - & 53.29 \\
\hline
\end{tabular}




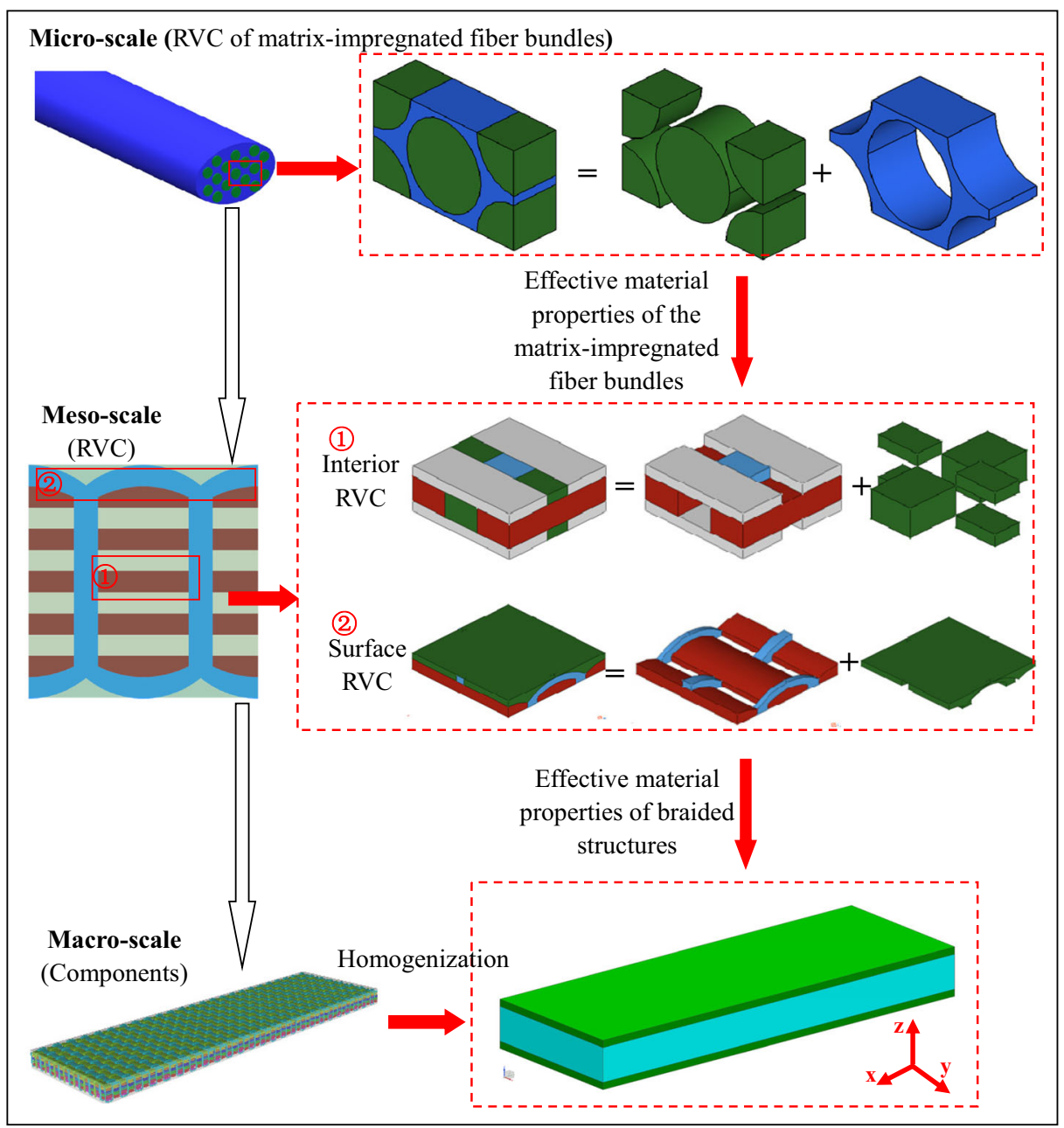

Fig. 7 Framework of multi-scale model of 3D orthogonal woven composite

periodic boundary condition ${ }^{22}$, the meso-scale materials properties of two solid RVCs are calculated.

Finally, meso-level data is delivered to the macro-scale homogeneous composite, and then the tensile properties of $3 \mathrm{D}$ orthogonal woven composites are simulated. Clearly, this macroscale finite element model can calculate the effective moduli and strength. Here, it scarcely evaluates the strain and stress elastic distribution of the whole field, as will be verified in a later section of this paper.

\section{Experimental Details}

3D orthogonal woven preforms are fabricated using the 3D woven machine at the Institute of Composite Materials of Tianjin Polytechnic University (China). Then epoxy matrix (TDE 86) 
is injected into 3D orthogonal woven preforms by the process of the resin transfer molding (RTM) to produce carbon/epoxy and carbon/Kevlar/epoxy composite specimens, as shown in Fig. 8. The specimen geometry and dimensions are depicted in Fig. 9a. In preparing the tensile samples, each side is affixed to an aluminum strengthening plate with the epoxy glue film to protect the specimen from damage that caused by the rigid test fixture during the test, as show in Fig. 9a.

For the tensile tests, the standard test methods ASTM D3039 are followed. All the tests are carried out by SHIMADZU AG-250KNE equipment in the room temperature. For validating the rationality of FEMM and MFEM, the digital image correlation technique (DIC, see Fig. 9b) is used to measure the full-field strain distribution of composite components subjected to tensile loading $[22,23]$. This technique is becoming an widely popular analytical tool in the field of structural mechanics owing to the low price and availability of imaging setup and correlation software. A key step is that the white speckle pattern on a matt black base coat is needed to paint onto the surface of the tensile specimens, as shown in Fig. 9c-d. Then the charge coupled device (CCD) camera is used to obtain grey scale images of the samples during loading. Noted that the measurement error of DIC is estimated less than $2 \%$. Each test result in this paper is the mean of three repeats.

\section{Results and Discussion}

After generating the predicted models with two types of methods, VFEM and MFEM, a commercial finite element software ABAQUS/Standard is employed to calculate the macromechanical properties of 3D orthogonal woven composites subjected to tensile loading. The primary goal of the development of voxel-based finite element method (VFEM) is to simulate the full-field strain and stress distribution. Furthermore, the analytical results of VFEM are compared to those of MFEM and DIC measurement to validate the accuracy of this model.

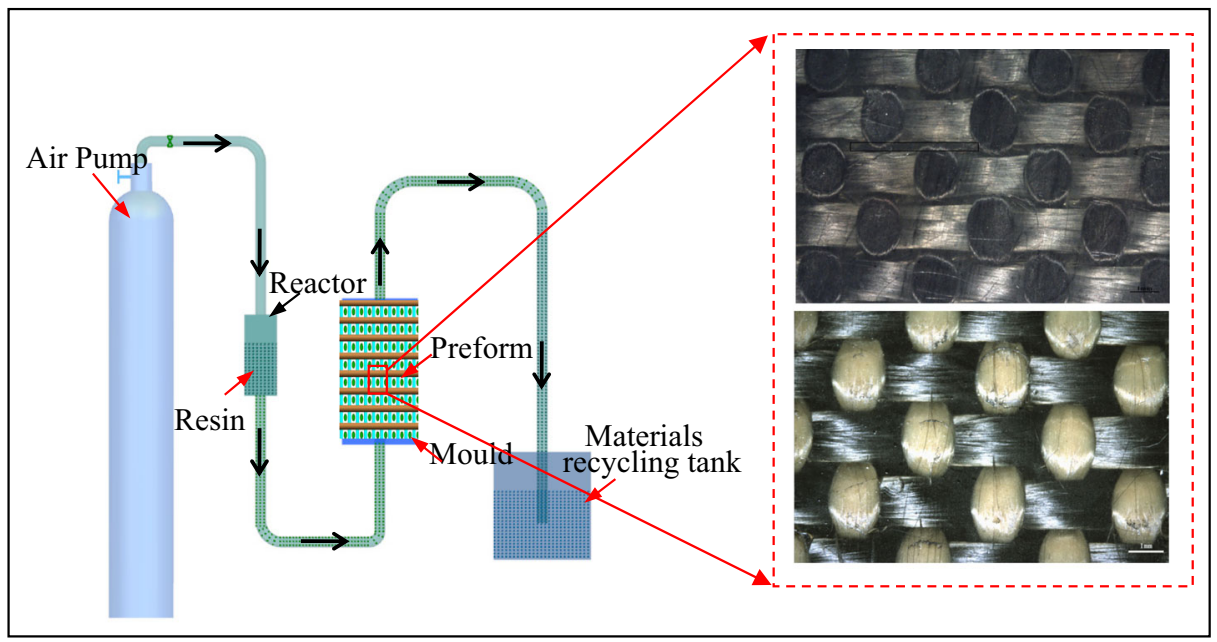

Fig. 8 The process of the resin transfer molding 


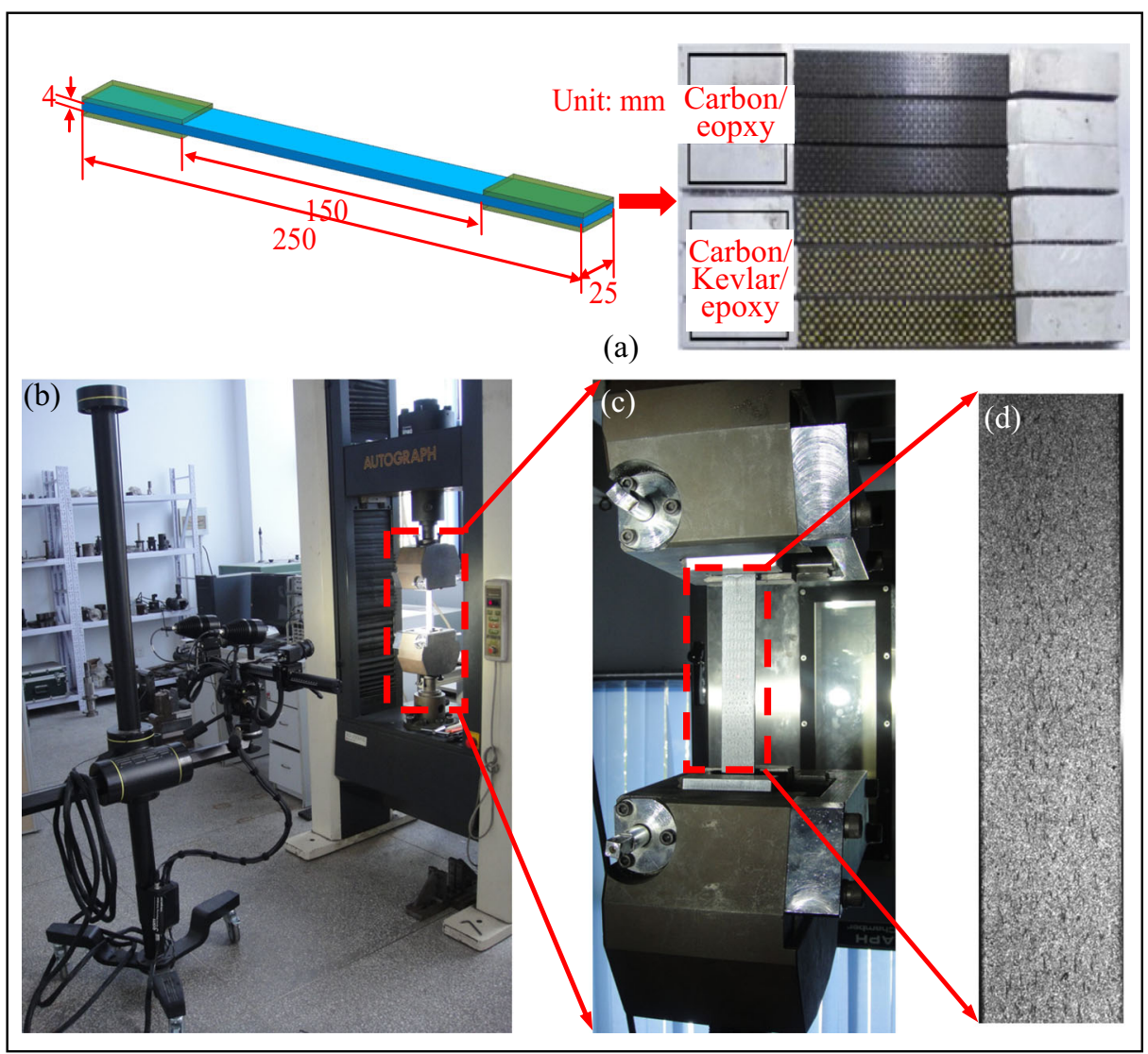

Fig. 9 Tensile test with digital image correlation setup

\subsection{Global Stress-Strain Curves}

Figure 10 shows the comparisons of the typical stress-strain curves between simulation and experiment. Clearly, the predicted stress-strain curve tendency of both VFEM and MFEM is essentially in accordance with the experimental data. The computed initial modulus is in good agreement with the experimental result. Also, it should be noted that the stress-strain curve of all the specimen exhibits significantly linear characteristics, indicating that both carbon/epoxy and carbon/Kevlar/epoxy 3D orthogonal woven composites are brittleness material. Moreover, as compared to carbon/epoxy composites, the failure strain of carbon/Kevlar/epoxy woven composites is higher, suggesting a excellent toughness properties.

Additionally, for carbon/epoxy composites, it is found that the max stress of voxel-based finite element method (VFEM, 937.66 MPa) exceeds slightly that of multi-scale finite element (MFEM, $892.36 \mathrm{MPa}$ ) by $5.08 \%$ and the experiment $(911.33 \mathrm{MPa})$ by $2.89 \%$. Also, for carbon/Kevlar/epoxy composites, the max stress of the experiment, VFEM and MFEM is 1020.01 MPa, 1034.35 MPa and 1007.05 MPa, respectively. For aforementioned results, it can be concluded that as compared to the multi-scale model, voxel-based model exhibits more precise predicting ability. 


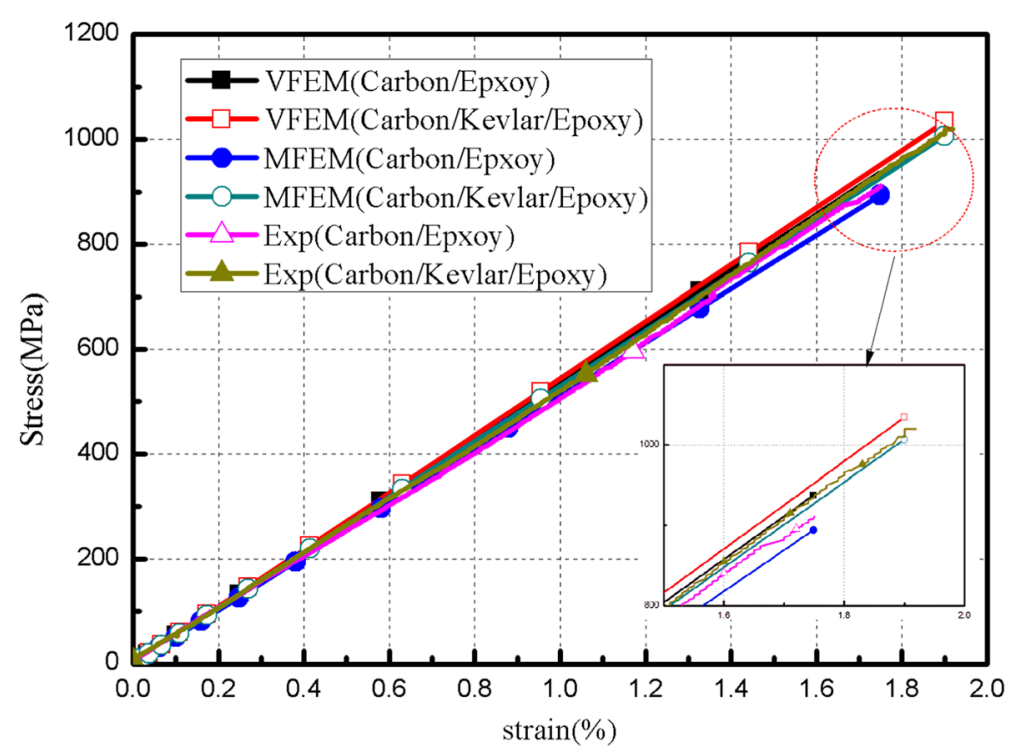

Fig. 10 Predicted and tested stress-strain curves

\subsection{Digital Image Correlation Validation}

Figure 11 displays the major strain contours along the longitudinal direction at the average strain level of $1.0 \%$. For both carbon/epoxy and carbon/Kevlar/epoxy 3D orthogonal woven composites, the predicted results obtained from VFEM and meso-scale solid model correlate the DIC image very excellently. However, unlike the above result, it is noticed that the numerical image from macro-scale solid model shows that the strain isolines are smooth and uniform. Not surprisingly, because the heterogeneity of the meso-structure is considered in both VFEM and meso-scale solid model. Furthermore, the predicted major strain fields by VFEM are somewhat regular than experimental one, attributable to the varying of the local structure parameters during the process of composite preparation. As for the graphs, the results of VFEM illustrate that the low strain concentrations exhibit the marked directivity. Combined with the result of meso-scale solid model, it can be concluded that the localized low strain areas are occurred in the $\mathrm{Z}$ yarns, whereas the localized high strain regions occur mainly in the resin rich zones. Moreover, for the surface low strain areas, the comparison between the predicted carbon/epoxy and carbon/Kevlar/epoxy 3D orthogonal woven composites are found of little difference, which can be attributed to the varying of the $\mathrm{Z}$ yarn areas on the surface. This result also be verified by the meso-scale geometric models in Fig. 3. Also, For both carbon/epoxy and carbon/Kevlar/epoxy 3D woven orthogonal composites, the observed fracture surface are relatively even, indicating the brittle rupture. This result is consistent with the obtained conclusion of the stress-strain curve as mentioned earlier.

In order to make a better interpretation of the tensile behaviors of 3D woven orthogonal composites, the predicted stress field contours of carbon/epoxy and carbon/Kevlar/epoxy structures are presented in Figs. 12 and 13, respectively. In Figs. 12a and 13a, the predicted result of VFEM fully captures the stress distribution of the whole field. It is interesting to see that the stress distributions have a close relationship with the yarn paths. Moreover, warp and Z 


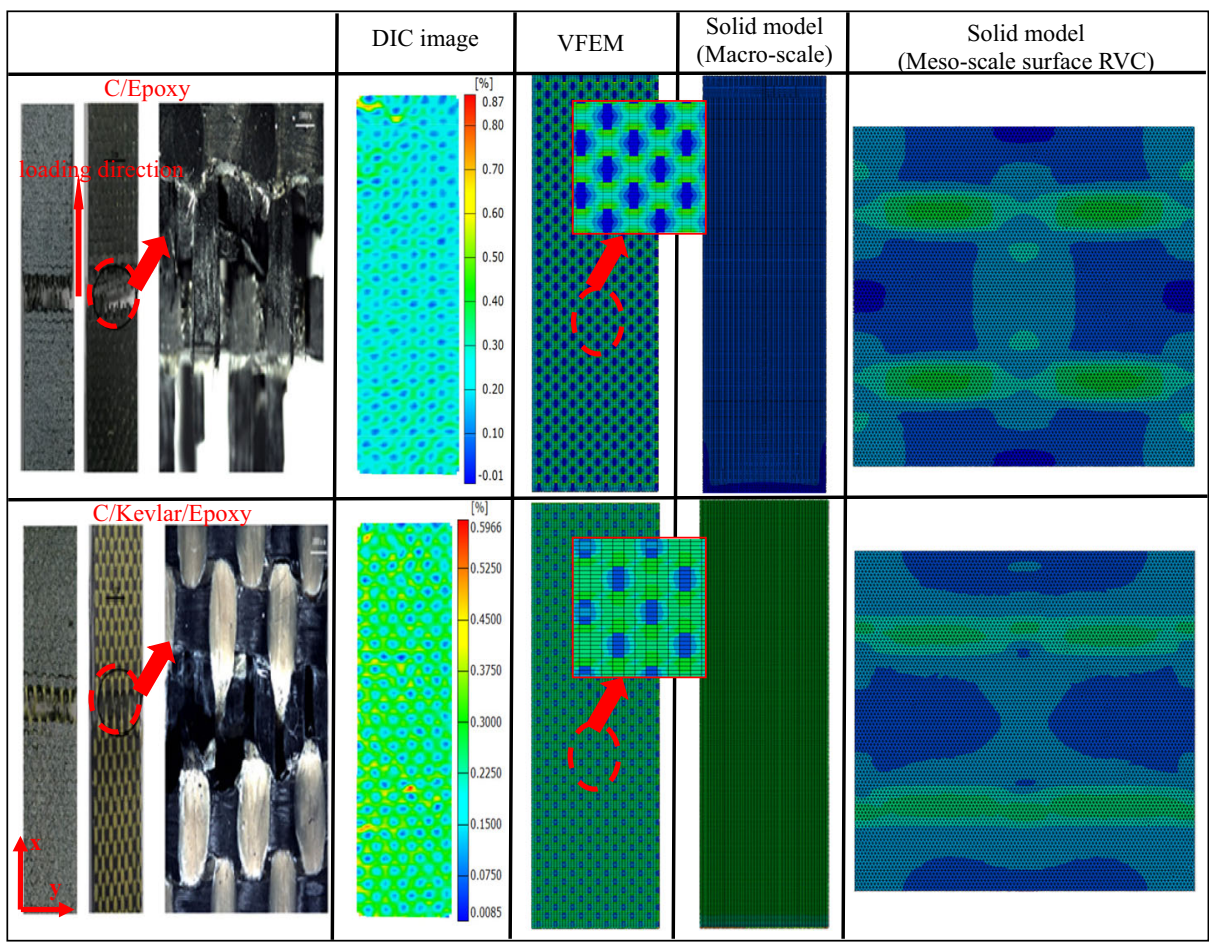

Fig. 11 Experimental and numerical surface major strain contours

yarn are observed to have very high stresses as compared to matrix and weft. As to the graphs in Figs. $12 \mathrm{~b}$ and 13b, macro-scale solid models with a homogeneous materials exhibit the smooth contours. Also, the highest stresses are located at the interior regions. This result is consistent with that of meso-scale solid models. This is because that warp are straight, and bear more loads.

\subsection{Effect of Voxel Mesh Convergence}

In this paper, three different voxel numbers through warp and thickness direction are chosen and the number of voxels $\left(N_{y}\right)$ through the thickness is fixed. Figures 14 and 15 show the warp strain and stress field of 3D carbon/epoxy orthogonal composites subjected to warp tensile loading, respectively. It can be clearly seen that the full-field strain and stress distribution of the $\mathrm{x}-\mathrm{y}$ plane has obvious difference. Moreover, the strain concentration zones are observed in all voxel meshes (white box in Fig. 14). This can be attributed to the added matrix elements between yarns, missing yarn elements and unsmooth link etc (see Fig. 6) as mentioned earlier. As to the graphs $15 \mathrm{a}$ and $15 \mathrm{~b}$ (white box), it also can be observed the stress concentration, while this phenomenon doesn't appear in the finest meshes (Fig. 15c). This is probably because that for that the mesh size is too coarse to well describe the yarn shape. Grail et al. [24] reported that the maximum edge length of the elements is a key parameters which influences the final mechanical behaviors, while the shorter edges are used only where it is needed to precisely remodel the geometry shape. The above analysis confirms that the mesh size effect plays an important role, which needs to a further study. 


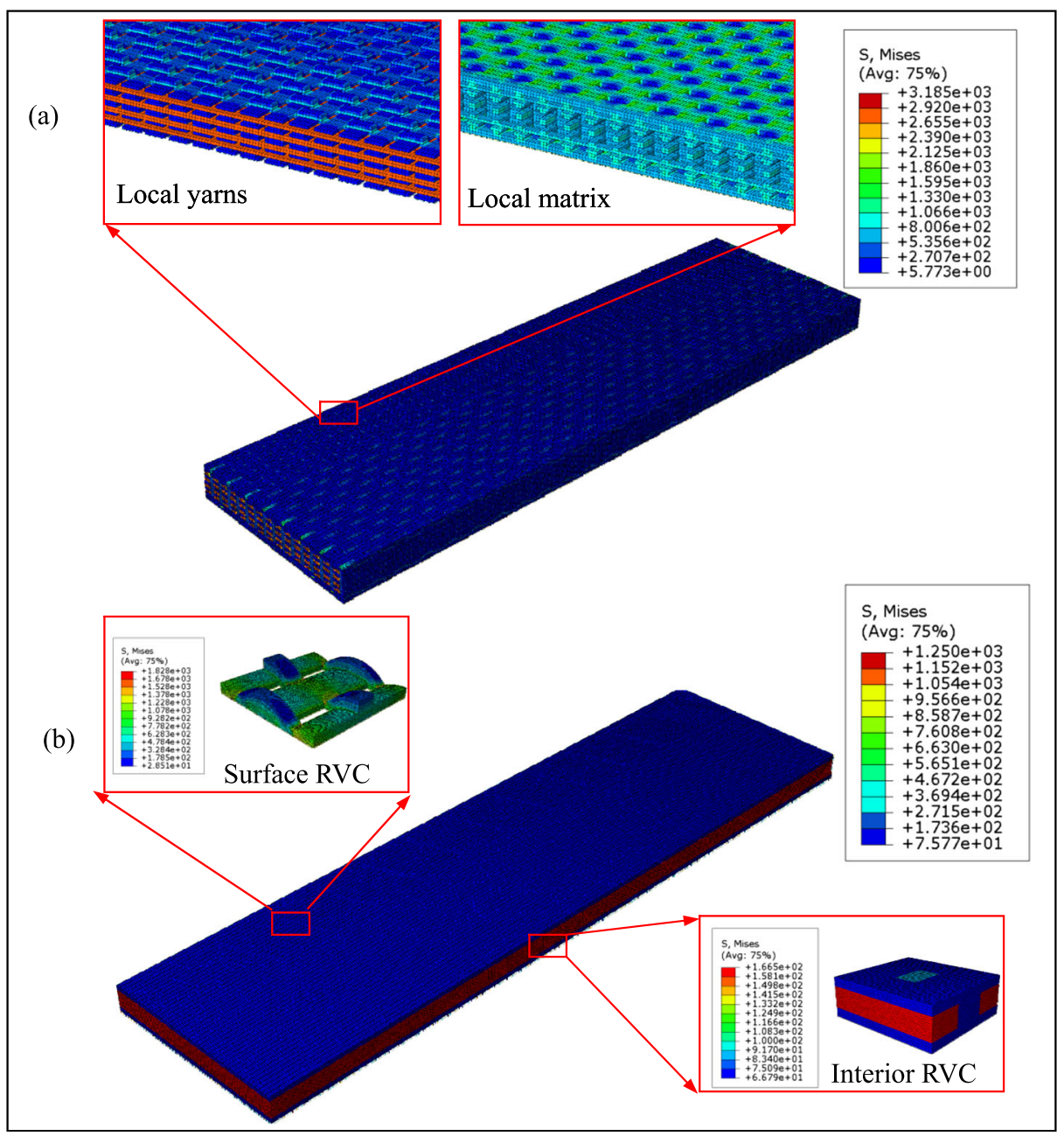

Fig. 12 Predicted strain field contours of 3D orthogonal woven composite, (a) Voxel-based finite element method, (b) Multi-scale finite element method

\subsection{General Discussion of the Prediction Model}

For afore-mentioned results, it can be concluded that the developed voxel-based finite element method (VFEM) is capable of accurately predicting the macroscopic elastic properties of 3D woven orthogonal composites when subjected to tensile loading. The ability of this model to obtain the stress-strain responses and the whole major strain distributions is clearly presented. The predicted major strain contours agree very well with the DIC experimental result, which is one of the contributions of this paper. Moreover, for VFEM, the discrete mesh process are simple and easily implemented into the ABAQUS software. More importantly, VFEM can reflect precisely the effect of the full-field fiber distribution, and probably extend to any textile structural composite and the arbitrarily shaped components. This is seen as an obvious advantage over many existing models in the previous literature. 


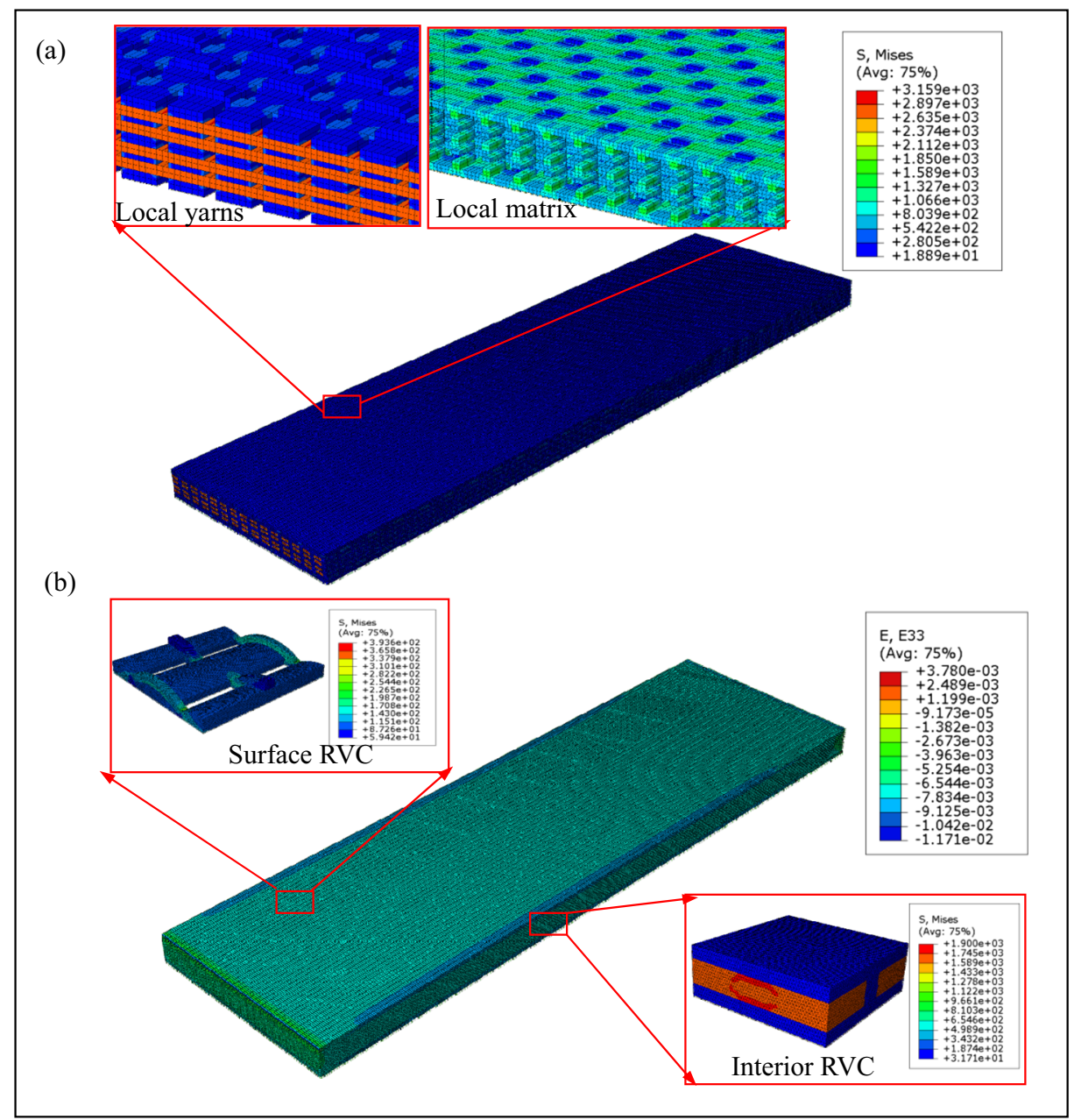

Fig. 13 Predicted stress field contours of 3D orthogonal woven composite, (a) Voxel-based finite element method, (b) Multi-scale finite element method

Although the calculated capability of VFEM has been shown, the development of this model is still in progress because there are a few of limitations with regards to its applicability. For example, stress concentrations are found at the step-like surfaces of the yarns, which may influence the damage prediction. Therefore, a further consideration may involve developing new mesh strategy and suitable damage model.

\section{Conclusions}

The main objective of this study is to develop voxel-based finite element method (VFEM) to predict the macro-mechanical properties of 3D woven orthogonal composites. For comparison, conventional multi-scale finite element method (MFEM) and digital image correlation (DIC) tests are carried out. Based on the detailed analysis, following conclusions are made: 


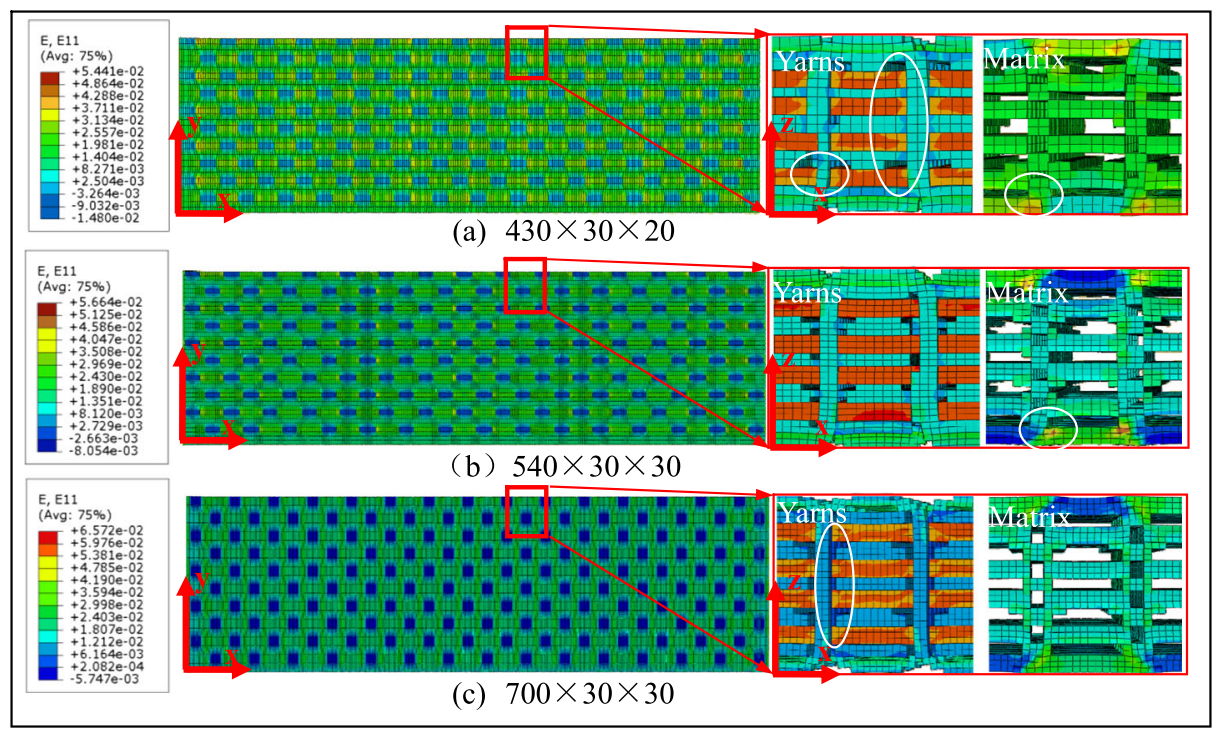

Fig. 14 Predicted strain field contours of voxel-based finite element method with different voxel mesh sizes

1) From the comparisons of the stress-strain curves, the initial modulus and the max stress, the calculated results of VFEM correlate the DIC image very excellently.

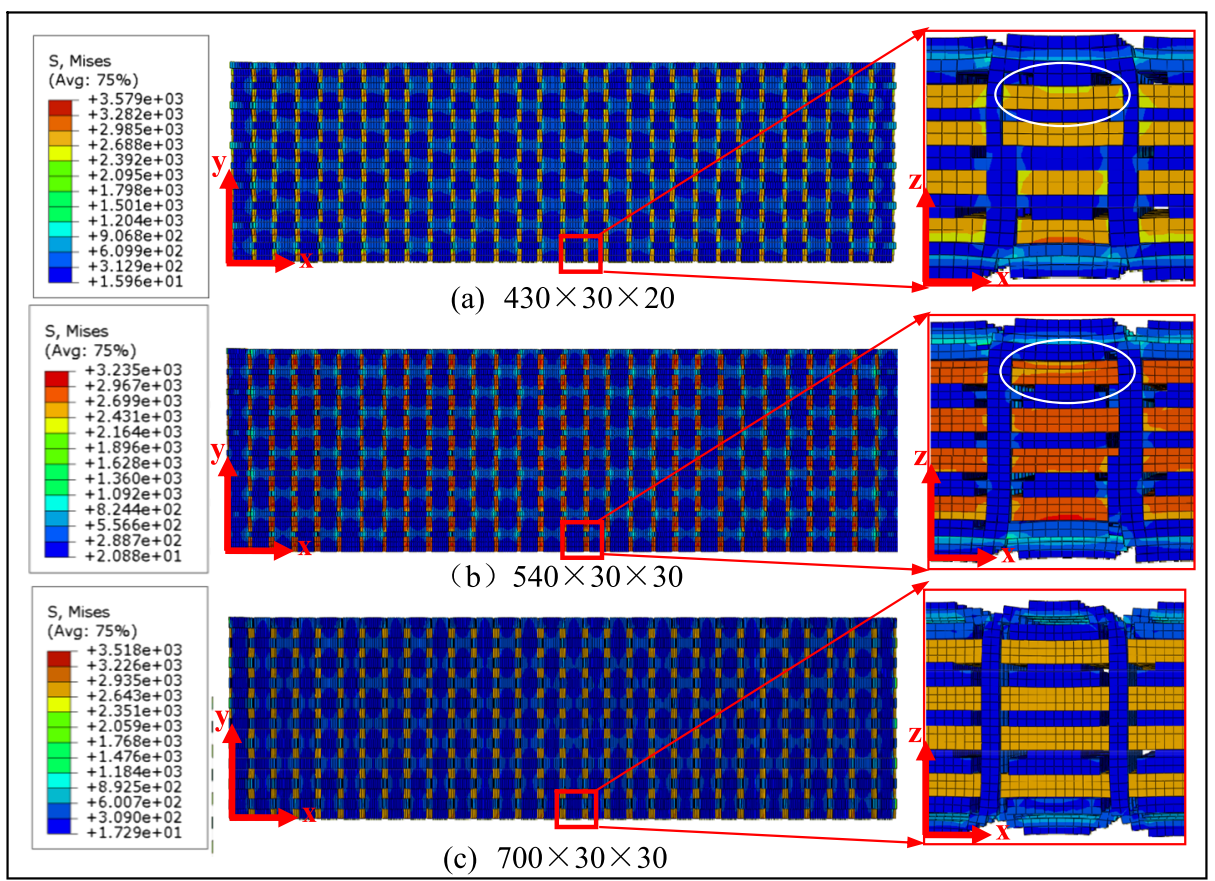

Fig. 15 Predicted stress field contours of voxel-based finite element method with different voxel mesh sizes 
2) Under warp tensile loading, both VFEM and DIC successfully obtain the full-field strain distribution, while MFEM hardly capture the local deformation in different areas. Also, the results of VFEM agree well with the experimental ones.

3) The developed voxel-based finite element model is capable of accurately calculating the macro-level elastic properties of 3D woven orthogonal composites when subjected to warp tensile loading. However, as for the future studies, it is desirable to continue with an effort to address some of its limitations, including the new mesh strategy and suitable damage model. These insights can be extremely helpful in solving any textile structural component with arbitrarily shaped.

Funding The authors gratefully acknowledge the financial support from Natural Science Foundation of China (No. 11702115), Natural Science Foundation of Jiangsu Province (P.R.China) (No. BK20170166) and Natural Science Foundation Central Universities (No. JUSRP11703).

\section{Compliance with Ethical Standards}

Conflict of Interest None declared.

Open Access This article is distributed under the terms of the Creative Commons Attribution 4.0 International License (http://creativecommons.org/licenses/by/4.0/), which permits unrestricted use, distribution, and reproduction in any medium, provided you give appropriate credit to the original author(s) and the source, provide a link to the Creative Commons license, and indicate if changes were made.

\section{References}

1. Hallal, A., Younes, R., Fardoun, F.: Review and comparative study of analytical modeling for the elastic properties of textile composites. Compos. Part. B-Eng. 50, 22-31 (2013)

2. Bilisik, K.: Multiaxis three-dimensional weaving for composites: a review. Text. Res. J. 82(7), 725-743 (2012)

3. Ansar, M., Wang, X.W., Zhou, C.W.: Modeling strategies of 3D woven composites: a review. Compos. Struct. 93(8), 1947-1963 (2011)

4. Lin, H., Ramgulam, R., Arshad, H., Clifford, M.J., Potluri, P., Long, A.C.: Multi-scale integrated modelling for high performance flexible materials. Comput. Mater. Sci. 65, 276-286 (2012)

5. Vanaershot, A., Cox, B.N., Lomov, S.V., et al.: Stochastic multi-scale modelling of textile composites based on internal geometry variability. Comput. Struct. 122, 55-64 (2013)

6. Deng, Y., Chen, X.H., Wang, H.: A multi-scale correlating model for predicting the mechanical properties of tri-axial braided composites. J. Reinf. Plast. Compos. 32(24), 1934-1955 (2012)

7. Kwon, Y.W., Kim, D.H., Chu, T.: Multi-scale modeling of refractory woven fabric composites. J. Mater. Sci. 41, 6647-6654 (2006)

8. Bacatteza, O., Aliabadi, M., Apicella, A.: Multi-scale failure analysis of plain-woven composites. J. Strain Anal. Eng. Des. 47(6), 379-388 (2012)

9. Lu, Z.X., Zhou, Y., Yang, Z.Y., Liu, Q.: Multi-scale finite element analysis of 2.5D woven fabric composites under on-axis and off-axis tension. Comput. Mater. Sci. 79, 485-494 (2013)

10. Dai, S., Cunningham, P.R.: Multi-scale damage modelling of 3D woven composites under uni-axial tension. Compos. Struct. 142, 298-312 (2016)

11. Bassam, E.S., Dmitry, I., Andrew, C.L., et al.: Multi-scale modelling of strongly heterogenerous 3D composite structures using spatial Voronoi tessellation. J. Mech. Phy. Solids. 88, 50-71 (2016)

12. Wang, Y.M., Sun, B.Z., Gu, B.H.: Multi-scale structure modeling of damage behaviors of 3D orthogonal woven composite materials subject to quasi-static and high strain rate compressions. Mech. Mater. 94, 1-25 (2016)

13. Zhang, D.T., Sun, Y., Wang, X.M., Chen, L.: Prediction of macro-mechanical properties of 3D braided composites based on fiber embedded matrix method. Compos. Struct. 134, 393-408 (2015)

14. Lomov, S.V., Verpoest, I., Cichosz, J., Hahn, C., Ivanov, D.S., Verleye, B.: Meso-level textile composites simulations: open data exchange and scripting. J. Compos. Mater. 48(5), 621-637 (2014) 
15. Dong, W.F., Xiao, J., Li, Y., et al.: Theoretical study on elastic properties of 2.5D braided composites. J NanJing Univ. Aeronaut. Astronaut. 37(5), 659-663 (2005)

16. Huang, Z.M.: Abridging model prediction of the ultimate strength of composite laminates subjceted to biaxial loads. Compos. Sci. Technol. 64, 395-448 (2004)

17. Vanaerschot, A., Cox, B.N., Lomov, S.V., Vandepitte, D.: Experimentally validated stochastic geometry description for textile composite reinforcements. Compos. Sci. Technol. 122, 122-129 (2016)

18. Paterl, D.K., Waas, A.M.: Damage and failure modelling of hybrid three-dimensional textile composites: a mesh objective multi-scale approach. Phil. Trans. R. Soc. A. 374, 1-31 (2016)

19. Doitrand, A., Fagiano, C., Irisarri, F.X., Hirsekorn, M.: Comparison between voxel and consistent mesoscale models of woven composites. Compos Part A-App. 73, 143-154 (2015)

20. Isart, N., Said, B.E., Hallett, S.R., et al.: Internal geometric modelling of 3D woven composites: a comparison between different approaches. Compos. Struct. 132, 1219-1230 (2015)

21. Zhang, C., Xu, X.W.: Finite element analysis of 3D braided composites based on three unit-cells models. Compos. Struct. 98, 130-142 (2013)

22. Koerber, H., Xavier, J., Camanho, P.P., Essa, Y.E., Martín de la Escalera, F.: High strain rate behavior of 5harness-satin weave fabric carbon-epoxy composite under compression and combined compression-shear loading. Int. J. Solids Struct. 54, 172-182 (2015)

23. Grammond, G., Boyd, S.W., Dulieu-Barton, J.M.: Evaluating the localised through-thickness load transfer and damage initiation in a composite joint using digital image correlation. Compos. Part A-App. S. 61, 224 234 (2014)

24. Grail, G., Hirsekorn, M., Wendling, A., Hivet, G., Hambli, R.: Consistent finite element mesh generation for meso-scale modeling of textile composites with preformed and compacted reinforcements. Compos. Part AApp. 55, 143-151 (2013) 